\title{
La legislación rusa sobre el autogobierno local ${ }^{* *}$
}

\section{Russian Legislation on Local Self-Government}

RESUMEN

En este artículo se ofrece un estudio de las normas de la Constitución de la Federación de Rusia y de la legislación federal de Rusia sobre el autogobierno local. El artículo se centra en la naturaleza jurídica de las normas de la Constitución de Rusia, las principales decisiones del Tribunal Constitucional de la Federación de Rusia sobre la materia, y los trabajos de los principales científicos rusos. Se ha determinado la tendencia del desarrollo de la legislación rusa sobre el autogobierno local en los últimos años, incluso se ha analizado la reforma constitucional de 2020 en Rusia con respecto al autogobierno local. En la conclusión se señala que la regulación constitucional bastante detallada aún no garantiza el desarrollo rápido y efectivo del principio de descentralización del poder en el ámbito local. La legislación ordinaria, la jurisprudencia del Tribunal Constitucional y la situación política tienen mucha importancia en este proceso.

PALABRAS CLAVE

Constitución, autogobierno local, Rusia, reforma constitucional, fundamento del sistema constitucional.

\section{ABSTRACT}

This article offers a study of the norms of the Constitution of the Russian Federation and the federal legislation of Russia on the local self-government.

\footnotetext{
* Candidato de Ciencias Jurídicas. Docente en la Universidad de Rusia de la Amistad de los Pueblos "Peoples" Friendship University of Russia (RUDN University) (Instituto de Derecho, Departamento de Derecho Municipal). Moscú.Correos electrónicos: komleve1991@ gmail.com y komleve@mail.ru. oRCID: https://orcid.org/0000-0001-7246-8014.

** This paper has been supported by the RUDN University Strategic Academic Leadership

Recibido el 2 de abril del 2020, aprobado el 31 de agosto del 2020.

Para citar el artículo: La legislación rusa sobre el autogobierno local. En Revista Derecho del Estado, Universidad Externado de Colombia. N. . 48, enero-abril de 2021, 243-271.

DOI: https://doi.org/10.18601/01229893.n48.09
} Program. 
The article focuses on the legal nature of the norms of the Constitution of Russia, the main decisions of the Constitutional Court of the Russian Federation on this issue, and the works of leading Russian scientists. The tendency of the development of Russian legislation on the local self-government in recent years is determined; including the constitutional reform in Russia of 2020 regarding the local self-government is analyzed. In the conclusion it states that a detailed constitutional regulation does not guarantee the rapid and effective development of the principle of decentralization of power at the local level. The ordinary legislation, judicial practice of the Constitutional Court and the political situation are very important in this process.

\section{KEYWORDS}

Constitution, local self-government, Russia, constitutional reform, foundation of the constitutional system.

SUMARIO

Introducción. I. El autogobierno local en Rusia hasta la adopción de la Constitución de 1993. II. Los principios básicos de la regulación jurídica del autogobierno local en Rusia. A. El autogobierno local como una forma de ejercicio por parte del pueblo de su poder. B. El autogobierno local como el fundamento del sistema constitucional. C. Los órganos del autogobierno local en el sistema de las autoridades públicas en Rusia. D. El derecho a ejercer el autogobierno local. III. El capítulo 8, "El autogobierno local”, de la Constitución de Rusia. Conclusiones. Bibliografía

\section{INTRODUCCIÓN}

Actualmente, en ciencia jurídica, se presta mucha atención a la institución del autogobierno local ${ }^{1}$. Este último se considera uno de los fundamentos de un estado democrático, porque en gran parte es un fenómeno legal único. Su carácter único se muestra en varios aspectos, entre los cuales se encuentra la naturaleza de los órganos locales. Ellos son autoridades públicas, pero simultáneamente son los órganos más próximos al ciudadano.

En la institución del autogobierno local existe un enorme potencial democrático. Con el funcionamiento efectivo del autogobierno local, los ciudadanos de las respectivas entidades territoriales tienen la oportunidad de influir en

1 En el presente trabajo, la terminología correspondiente se utiliza de acuerdo con la legislación y la doctrina rusa. En particular, se utilizan los términos "el autogobierno local" (el sinónimo del cual es el término "la autonomía local"), "los órganos del autogobierno local" o "los órganos locales" (administraciones locales según la terminología en español). 
la resolución de problemas relacionados con su vida diaria, satisfaciendo al máximo sus necesidades e intereses. Dicha influencia se lleva a cabo, entre otras cosas, a través de la participación en la vida pública y política de la entidad territorial correspondiente.

Al mismo tiempo, para conseguir el funcionamiento efectivo del autogobierno local se requiere una regulación normativa adecuada, que presuponga un equilibrio en la distribución de poderes entre varias autoridades públicas.

Una de las características del autogobierno local es que las actividades de los órganos locales, incluidas sus competencias, dependen en gran medida de la regulación jurídica, llevada a cabo en los ámbitos nacional y regional. Y otra característica esencial es que no existe una "receta" única para garantizar su funcionamiento efectivo. En cada estado (e incluso dentro del marco de un estado) existen particularidades que de una forma u otra afectan el contenido de las normas jurídicas relevantes, así como los objetivos que enfrenta el legislador.

En este sentido, el estudio de la experiencia de varios estados en materia de regulación jurídica del autogobierno local es de indudable interés científico y tiene un valor práctico. La incorporación de ciertos mecanismos de regulación jurídica (teniendo en cuenta todas las ventajas y las desventajas de la regulación jurídica en otros estados) tiene un impacto positivo en el desarrollo de la legislación nacional sobre el autogobierno local.

La experiencia rusa de regulación jurídica del autogobierno local en cierto sentido es única: hace relativamente pocos años (en la primera mitad de la década de 1990), el régimen político en el país ha cambiado significativamente. Rusia se ha proclamado un estado democrático. Se tomó un curso hacia la descentralización y la distribución vertical del poder.

Y es evidente que en Rusia existen características esenciales históricas, políticas, legales, que influyen el desarrollo del autogobierno local.El legislador ruso tuvo en cuenta la experiencia de los estados extranjeros (principalmente europeos) en materia de regulación jurídica del autogobierno local, así como su propia experiencia histórica.

La base jurídica del autogobierno local en Rusia, como es lógico, son las normas de la Constitución de Rusia. En el presente trabajo se analizan las normas de la Constitución de Rusia, directa o indirectamente dedicadas al autogobierno local, las normas de la legislación ordinaria, adoptadas sobre la base de las disposiciones de la Constitución de Rusia, la jurisprudencia del Tribunal Constitucional de la Federación de Rusia sobre nuestra materia y los estudios de los científicos rusos.

El análisis de las normas constitucionales y la práctica de su implementación permite determinar la naturaleza jurídica del autogobierno local en el marco del sistema legal ruso, así como las perspectivas de un mayor desarrollo de esta institución. 
I. EL AUTOGOBIERNO LOCAL EN RUSIA HASTA

LA ADOPCIÓN DE LA CONSTITUCIÓN DE 1993

A lo largo de casi toda su historia, Rusia ha sido un estado altamente centralizado. En ciertos períodos de su desarrollo los gobernadores iniciaron la implementación de unas reformas, en cierta medida dirigidas a la descentralización del poder.

Algunos autores rusos afirman que el autogobierno local en Rusia surgió durante la formación y el desarrollo del sistema comunal entre los eslavos ${ }^{2}$.

La opinión bastante popular es que el autogobierno local en Rusia surgió en el momento en que la tradición de resolver los problemas importantes de la vida pública en un consejo (veche) estaba muy extendida. Los ejemplos más ilustrativos de la democracia veche son las repúblicas de Nóvgorod y Pskov. Como señalan N. A. Ignatyuk y A. V. Pavlushkin, "las primeras ideas sobre la independencia social como una base del poder provienen de la República de Nóvgorod"’.

Sin embargo, los ejemplos anteriores del autogobierno comunitario se refieren a los siglos x y XI. Es obvio que desde entonces el sistema sociopolítico ha cambiado tanto que en actualidad no se puede hablar de una continuidad significativa.

Más indispensable, la cuestión de la necesidad de reformar el autogobierno local y la gobernanza regional en Rusia se planteó en el siglo XIx, cuando varias figuras políticas y públicas propusieron conceptos para el desarrollo posterior del Estado en esta dirección ${ }^{4}$.

También coincidimos con V. I. Vasíliev cuando afirma que "el autogobierno local en el sentido actual generalmente aceptado se introdujo en Rusia en 1864 después de la reforma campesina de 1861, y lógicamente acompañó esta reforma, que dio libertad civil a la parte significativa de la población rural de Rusia. Todos los intentos previos de establecer el autogobierno local en Rusia sobre una base democrática (incluidos los de Pedro i y Ekaterina II) no pudieron dar efectos bajo la política de la servidumbre. La esclavitud de los campesinos impidió la conversión de privilegios nobles de clase en derechos civiles [...]. Tan pronto como se rompieron los lazos de esclavitud, una reorganización del autogobierno local sobre nuevas bases se hizo inevitable" 5 .

2 Postovoi, N. V. Mestnoe samoupravlenie. Istoriya, teoriya, praktika [Local Selfgovernment. History, theory, practice]. Moscow, Advertising and publishing center «Fedorov», 1995, p. 8 (en ruso).

3 Ignatyuk, N. A., Pavlushin, A. V. Munitsipal'noe pravo. Uchebnoe posobie. [Municipal law. Study guide]. Moscow, Yustitsinform, 2007, p. 64 (en ruso).

4 EREMYAN, V. V. Teoreticheskie obosnovaniya neobhodimosti reformirovaniya mestnogo samoupravleniya i regional'nogo upravleniya $v$ Rossii vo vtoroj polovine XIX veka [The theoretical grounds of the local's and regional's government reform in the second half of the XLXth century in Russia]. RUDN Journal of Law, 2000, n. ${ }^{\circ}$, p. $49-53$ (en ruso). 
No obstante, incluso después de la liberación de los campesinos y las reformas correspondientes de la administración pública en la década de 1860 , los gobiernos locales siguieron siendo muy dependientes del Estado. Una rápida transición al establecimiento de la independencia de los gobiernos locales no sucedió y apenas podría suceder. En algunas regiones de Rusia (en particular, en Siberia), las disposiciones de la reforma de la década de 1860 no se implementaron completamente hasta 1917, después de lo cual las ideas de reforma perdieron su relevancia en relación con la Revolución, la guerra civil y el establecimiento del régimen soviético.

A este respecto apenas se puede concluir que durante la época presoviética en Rusia hubo desarrollo pleno y universal del autogobierno local como una institución jurídica en su comprensión actual.

Después de la Revolución de 1917 y el establecimiento de la "dictadura del proletariado urbano y rural" (el artículo 9 de la Constitución de la RSFSR de 1918), el país pasó a una etapa de desarrollo completamente nueva.

Cabe mencionar que el modelo soviético de organización de la autoridad pública se basó en gran medida en la negación de la experiencia previa (principalmente por razones ideológicas). Como señala V. I. Vasíliev, "los bolcheviques, que tomaron el poder como resultado de la Revolución de octubre de 1917, comenzaron a construir su estado, confiando en la tesis marxista-leninista sobre la necesidad de un colapso radical de la vieja máquina del Estado. Los gobiernos locales, como instituciones del Estado burgués, fueron objeto de abrogación, aunque no de inmediato" . $^{\circ}$

Los primeros documentos jurídicos postrevolucionarios indicaban que los Sóviets de los Diputados de Obreros, Soldados y Campesinos son completamente independientes en asuntos locales, pero siempre actúan de acuerdo con los decretos y resoluciones tanto del poder central soviético como de los demás (sóviets regionales, provinciales etc.), de los cuales ellos forman parte. Esas disposiciones también se encontrarían en las posteriores constituciones soviéticas.

A nuestro entender el autogobierno local es una institución jurídica especial, cuyo desarrollo completo solo es posible en las condiciones del desarrollo democrático del Estado; en primer lugar, estamos hablando de aspectos como la primacía de los derechos humanos y las libertades en el sistema de valores del Estado, así como del reconocimiento del derecho de los ciudadanos a participar en la gestión de los asuntos públicos.

En el marco del modelo soviético de administración pública, el concepto de democracia se entendía de manera bastante peculiar. Es cierto que los

5 VAsILIEv, V. I. Munitsipal'noe pravo Rossii. Uchebnik. Vtoroe izdanie. [Municipal law of Russia. Textbook. Second edition.]. Moscow, Yustitsinform, 2012, p. 70 (en ruso).

6 Vasiliev, V. I. Munitsipal'noe pravo Rossii. Uchebnik. Vtoroe izdanie. [Municipal law of Russia. Textbook. Second edition.]. Moscow, Yustitsinform, 2012, p. 90 (en ruso). 
valores democráticos no podían competir con el componente ideológico del Estado soviético, que negaba todo lo ajeno al socialismo, todo lo burgués (este concepto a menudo se interpretaba de manera bastante amplia).

A pesar de la proclamación del principio de que todo el poder en el centro y en las localidades pertenece a los Sóviets de los Diputados de Obreros, Soldados y Campesinos, la Federación de Rusia y, posteriormente, la URSS fueron estados altamente centralizados de facto e incluso de jure.

Las actividades de las autoridades locales se controlaban por las autoridades superiores. En particular, el artículo 146 de la Constitución de la URss de 1977 (la última Constitución adoptada durante la época soviética) estableció que las autoridades locales (sóviets locales de Diputados Populares) "hacen cumplir las decisiones de los órganos estatales superiores y dirigen las actividades de los sóviets de los diputados populares del nivel inferior". El artículo 150 de la Constitución de la uRss de 1977 estableció que "los órganos ejecutivos y administrativos de los sóviets locales son directamente responsables ante el sóviet que los eligió y ante el órgano ejecutivo y administrativo superior".

Por todo ello podría concluirse que la Constitución soviética del año 1977 (así como las demás) fijó la jerarquía de autoridades, en la cual las autoridades inferiores estuvieron realmente y legalmente subordinadas y fueron responsables ante las autoridades superiores.

Considerando todo lo anterior, hay que señalar que el estado ruso actual es el sucesor de dos tipos de estados muy diferentes desde el punto de vista jurídico e ideológico: la URSs por un lado, la Rusia prerrevolucionaria por el otro.

Por supuesto, dada la cronología histórica, desde el punto de vista de la legislación, de cultura jurídica de los ciudadanos y de los líderes de varios niveles, de organización institucional y administrativa, la Rusia actual es en mayor medida el sucesor del Estado del período soviético.

Es innegable que las características históricas mencionadas deben tenerse en cuenta en el estudio y el análisis de la naturaleza jurídica de las normas de la actual Constitución de Rusia, incluidas las normas dedicadas a la regulación del autogobierno local.

\section{LOS PRINCIPIOS BÁSICOS DE LA REGULACIÓN}

JURÍDICA DEL AUTOGOBIERNO LOCAL EN RUSIA

La Constitución de la Federación de Rusia, adoptada el 12 de diciembre de 1993, marcó el comienzo de un sistema de organización de la autoridad pública fundamentalmente nuevo para Rusia. Este sistema es significativamente diferente de los que fueron establecidos en las constituciones adoptadas en la URSS.

La Constitución actual de Rusia por primera vez reconoce y garantiza el autogobierno local en la forma en que esta institución se entiende en la legislación occidental (principalmente europeo). 
El análisis de la legislación rusa, de la jurisprudencia del Tribunal Constitucional de la Federación de Rusia y de la doctrina científica muestra que la Constitución y, como consecuencia, la legislación ordinaria consideran la institución del autogobierno local desde diferentes puntos de vista.

\section{A. El autogobierno local como una forma de ejercicio por parte del pueblo de su poder}

La Constitución de Rusia considera al autogobierno local una forma (método) del ejercicio de poder por parte del pueblo. Esta conclusión se desprende de las disposiciones del artículo 3 de la Constitución de Rusia, de conformidad con las cuales:

a. el titular de la soberanía y la única fuente de poder en la Federación de Rusia es su pueblo multinacional; y

b. el pueblo ejerce su poder directamente, así como a través de los órganos del poder estatal y de los órganos del autogobierno local.

Las normas del artículo 3. ${ }^{\circ}$ de la Constitución de Rusia se desarrollaron en la ley básica en materia del autogobierno local, o sea, la Ley Federal de del 6 de octubre de 2003 n. ${ }^{\circ} 131-\mathrm{FZ}$ "Sobre los principios generales de organización del autogobierno local en la Federación de Rusia". Esta ley sustituyó a la anterior Ley Federal del 28 de agosto de 1995 n. ${ }^{\circ}$ 154-FZ, según la cual los sujetos de la Federación tenían poderes significativamente mayores en la regulación jurídica del autogobierno local que bajo la actual legislación rusa.

Según el artículo $1 .^{\circ}$ de la Ley Federal n. ${ }^{\circ} 131-\mathrm{FZ}$, el autogobierno local se entiende como "una forma de ejercicio por parte del pueblo de su poder, que garantiza (dentro de los límites establecidos por la Constitución de la Federación de Rusia, las leyes federales y, en los casos establecidos por las leyes federales, las leyes de los sujetos de la Federación) a la población la resolución independiente y bajo su propia responsabilidad de los asuntos de importancia local, que se realiza directamente y (o) a través de los órganos del autogobierno local y se basa en los intereses de la población, teniendo en cuenta tradiciones históricas y otras tradiciones locales".

Dicha definición de la ley federal rusa no es igual que, por ejemplo, la de La Carta Europea de Autonomía Local ${ }^{7}$, que define el autogobierno local como "el derecho y la capacidad efectiva de las Entidades locales de ordenar y gestionar una parte importante de los asuntos públicos, en el marco de la Ley, bajo su propia responsabilidad y en beneficio de sus habitantes".

7 Al mismo tiempo, la Carta Europea de la Autonomía Local forma parte del sistema legal de la Federación de Rusia en virtud del artículo 15 de la Constitución de Rusia, ya que ha sido ratificada según el procedimiento establecido. 
La Carta Europea de Autonomía Local se centra en los derechos de las entidades locales, mientras que la Constitución rusa y la legislación ordinaria enfatizan que el autogobierno local es una de las formas de realización del poder que pertenece al pueblo.

Es decir, la Constitución rusa y la legislación ordinaria consideran el autogobierno local como un mecanismo especial por el cual la población, independientemente y bajo su propia responsabilidad, resuelve cuestiones de importancia local dentro de los límites establecidos por la ley.

Al mismo tiempo, el artículo 3 de la Constitución rusa consagra dos formas en que el pueblo ejerce su poder en el ámbito local: el ejercicio directo de su poder y el ejercicio de su poder a través de los órganos locales.

El capítulo 5 de la Ley Federal n. ${ }^{\circ} 131-\mathrm{FZ}$ esclarece en qué formas el pueblo ejerce directamente su poder, indicando entre ellas referéndum local, elecciones locales, votación para revocar a un diputado, votación sobre cuestiones de cambio de los límites de territorios locales, iniciativa legislativa de los ciudadanos, audiencias públicas y otras.

El procedimiento para ejercer el poder a través de los órganos locales también está regulado por la Ley Federal n. $131-\mathrm{FZ}$, que consagra los tipos de los órganos locales, el procedimiento para su formación, sus competencias, los límites para controlar su actividad por parte del estado, la responsabilidad de los órganos locales y otras cuestiones.

Sin embargo, debe reconocerse que las mencionadas contradicciones en el enfoque para definir el concepto del autogobierno local (entre las disposiciones de la Carta Europea de Autonomía Local por un lado y la legislación nacional de Rusia por el otro) no afectan conceptualmente la comprensión de la naturaleza jurídica del autogobierno local en los sistemas jurídicas de Rusia y de otros estados extranjeros (principalmente europeos).

Tanto en Rusia como en otros estados la población local participa en la resolución de problemas de importancia local a través de los órganos locales relevantes, que ejercen sus poderes de conformidad con una ley correspondiente.

La característica del modelo ruso es que la Constitución enfatiza la fuente ("propietario") del poder que se ejerce en el ámbito local, que es el pueblo.

\section{B. El autogobierno local como el fundamento del sistema constitucional}

Desde el punto de vista de los valores constitucionales, la Constitución rusa reconoce el autogobierno local como uno de los fundamentos del sistema constitucional.

Que el autogobierno local en Rusia se considere uno de los fundamentos del sistema constitucional está establecido en el artículo 12 de la Constitución de Rusia, según el cual "el autogobierno local está reconocido y garantizado en la Federación de Rusia. El autogobierno local dentro de sus poderes es independiente". 
A su vez, el artículo 12 de la Constitución de Rusia se encuentra en el capítulo 1, "Los fundamentos del sistema constitucional", de la Constitución. $\mathrm{Y}$ eso tiene ciertas consecuencias legales importantes, especialmente en el contexto de la reforma constitucional, propuesta por el presidente Putin $^{8}$ y realizada en Rusia en marzo-julio de 2020, de la que trataremos más adelante.

De acuerdo con el artículo 16 de la Constitución de Rusia, las disposiciones del capítulo 1 de la Constitución son los fundamentos del sistema constitucional de la Federación de Rusia y no pueden ser reformados, excepto en la forma establecida por la Constitución.

Tal forma (de la reformación del capítulo 1 de la Constitución) se establece en el artículo 135 de la Constitución de Rusia, según el cual las disposiciones del capítulo 1, "Los fundamentos del sistema constitucional", del capítulo 2, "Los derechos y las libertades del hombre y del ciudadano", y del capítulo 9, "Las reformas constitucionales y la revisión de la Constitución", no pueden ser reformadas y revisadas por la Asamblea Federal (el Parlamento).

A diferencia de los capítulos 3-8 de la Constitución de Rusia, cuyas reformas pueden realizarse casi en el mismo orden en que se adoptan las Leyes Constitucionales Federales (Las leyes orgánicas diríamos con la terminología jurídica en español), las reformas de los capítulos 1,2 y 9 de la Constitución requieren la adopción de una nueva Constitución, cuyo proyecto debe ser preparado por un órgano especial: la Asamblea Constitucional. La Asamblea Constitucional se convoca de la manera prescrita por una ley constitucional federal especial (dicha ley aún no ha sido adoptada en la Federación de Rusia). Cuando se desarrollare un proyecto de nueva Constitución, este último se aprueba por 2/3 de los votos de los miembros de la Asamblea Constitucional o se somete a una votación popular (de facto un referéndum federal).

Por tanto, para reformar las disposiciones del capítulo 1 de la Constitución de Rusia se requiere la adopción de una nueva Constitución, cuyo desarrollo y aprobación es bastante complicado y se realiza en varias etapas. Algunos de los mecanismos de este procedimiento requieren una regulación legislativa adicional, sin embargo dicha regulación aún no se ha implementada en la legislación rusa.

En consecuencia, parece que actualmente no hay presuposiciones para que dicho procedimiento se inicie en el futuro próximo. Incluso a pesar de las enmiendas constitucionales propuestas por el presidente de Rusia en enero de 2020 y adoptadas en julio de 2020, que son muy significativas y bastante numerosas, la nueva Constitución no ha sido adoptada, ya que las enmiendas no afectan los capítulos 1, 2 y 9 de la Constitución rusa. Sin embargo, como lo demuestra la experiencia del constitucionalismo soviético y ruso, mucho depende no solo de las razones legales, sino por excelencia de la situación política. 
Además del hecho de que las disposiciones del capítulo 1 (y, por tanto, el artículo 12 sobre las garantías de la independencia del autogobierno local) no pueden reformarse en el marco de la Constitución actual de Rusia, el reconocimiento del autogobierno local como uno de los fundamentos del sistema constitucional tiene otra importancia práctica: de conformidad con el artículo 16 de la Constitución de Rusia, ninguna otra disposición de la Constitución puede contradecir los fundamentos del sistema constitucional de la Federación de Rusia.

Como consecuencia, las normas del capítulo 1 de la Constitución de Rusia no solo tienen un estatus especial en términos del procedimiento para reformación de la Constitución, sino que también tienen prioridad en caso de inconsistencias entre varias normas de la Constitución. Esto, en particular, significa que si se reforman los demás capítulos de la Constitución (por ejemplo, el capítulo 8 "El autogobierno local"), dichos cambios deben cumplir con el artículo 12 de la Constitución.

Por todo ello, la Constitución de Rusia reconoce el estatus legal especial del autogobierno local y la importancia especial de las garantías de la independencia del autogobierno local. El reconocimiento de tal estatus especial implica el reconocimiento del estatus especial de los órganos del autogobierno local en el sistema de las autoridades públicas (más sobre esto en adelante).

Basado en dichas normas de la Constitución de Rusia, la profesora E. S. Shugriná señala que el autogobierno local en Rusia es un principio fundamental de organización del poder, que junto con el principio de separación de poderes (la división horizontal de poderes) define un sistema de gestión (la división vertical de poderes) ${ }^{9}$.

El profesor V. I. Vasíliev afirma que "la importancia del autogobierno local como uno de los fundamentos del sistema constitucional se expresa más claramente en que junto con otras instituciones constitucionales encarna las ideas democráticas del nuevo estado ruso" 10 .

Lo anterior nos permite concluir que el autogobierno local es reconocido por la Constitución de Rusia como uno de los principios básicos de organización de la autoridad pública en Rusia.

\section{Los órganos del autogobierno local en el sistema de las autoridades públicas en Rusia}

El artículo 11 de la Constitución de la Federación de Rusia determina que el poder estatal en Rusia se ejerce en los ámbitos federal y regional por los órganos pertinentes a los que se refiere el artículo 11 de la Constitución.

9 ShUGrina, E. S. Ponyatie mestnogo samoupravleniya kak konstitutsionnoj tsennosti [The Concept of Local Self-government as a Constitutional Value]. Yuridicheskij mir [Juridical world], 2016, n. ${ }^{\circ}$ 2. Disponible en http://lawinfo.ru/catalog/contents-2016/juridicheskij-mir/2/ (en ruso).

10 Vasiliev, V. I. Munitsipal'noe pravo Rossii. Uchebnik. Vtoroe izdanie. [Municipal law of Russia. Textbook. Second edition.]. Moscow, Yustitsinform, 2012, p. 45 (en ruso). 
En particular, en el campo federal el poder estatal lo ejercen presidente de la Federación de Rusia, la Asamblea Federal (el Consejo de la Federación y la Duma Estatal), el Gobierno de la Federación Rusa y los tribunales de la Federación de Rusia.

De acuerdo con el artículo 12 de la Constitución de Rusia, los órganos del autogobierno local no forman parte del sistema de los órganos del poder estatal.

Esto significa que la Constitución de Rusia distingue las autoridades públicas entre las estatales y las locales, y establece que el poder estatal mismo se ejerce por los órganos federales y regionales; en el ámbito local, los ciudadanos ejercen su poder a través de los órganos locales independientes, que no constituyen una jerarquía con los órganos estatales.

En la doctrina jurídica rusa no existe una opinión única sobre la norma del artículo 12 de la Constitución rusa. El profesor de la Universidad Estatal de Moscú S. A. Avakyán afirma que esta norma puede subentender la oposición de los órganos del autogobierno local al Estado. El profesor Avakyán señala que por el momento el problema se ha resuelto de acuerdo con la doctrina de la Constitución Viviente; sin embargo, a largo plazo es imposible apoyarse únicamente en la interpretación de las normas de la Constitución por parte del Tribunal Constitucional, por lo que la cuestión de adoptar una nueva Constitución (teniendo en cuenta otras disposiciones problemáticas de la Constitución rusa) es bastante actual ${ }^{11}$.

Sin embargo, coincidimos con el presidente del Tribunal Constitucional de la Federación de Rusia V. D. Zorkin cuando afirma que la distinción entre las autoridades públicas (estatales y locales) no significa su oposición entre sí ${ }^{12}$. El desarrollo del instituto del autogobierno local después de la adopción de la Constitución de Rusia de 1993 no indica la oposición de los órganos del autogobierno local a los órganos del poder estatal.

La distinción entre las autoridades estatales y locales fue hecha principalmente debido a que los órganos locales son inherentemente más cercanos a la población que las autoridades estatales. Como señala el magistrado del Tribunal Constitucional de la Federación de Rusia N. S. Bóndar, “el autogobierno local es un tipo especial de la autoridad pública, que se implementa sobre la base de una combinación de los principios estatales y no estatales (comunes)"13.

11 AvaKYán, S.A. Modernizatsiya publichno-politicheskikh otnosheniy i konstitutsionnoe reformirovanie: problemy i perspektivy [Modernization of public and political relationships and constitutional reformation: issues and prospects]. Konstitutsionnoe i munitsipal'noe pravo [Constitutional and Municipal law], 2019, n. ${ }^{\circ}$ 9, p. 3-6. Disponible en http://lawinfo.ru/catalog/ contents-2019/konstitucionnoe-i-municipalnoe-pravo/9/ (en ruso).

12 ZorkIn, V. D. Kommentariy k Konstitutsii Rossiyskoy Federatsii. Vtoroe izdanie (peresmotrennoe). [Commentary on the Constitution of the Russian Federation. Second edition (revised)]. Edited by V.D. Zorkin. Moscow, Norma, 2011, p. 150 (en ruso).

13 Voto particular del Magistrado del Tribunal Constitucional de la Federación de Rusia. Sentencia del Tribunal Constitucional de la Federación de Rusia n. ${ }^{\circ} 30-\mathrm{P}$ del $1 .^{\circ}$ de diciembre de 2015. 
Es evidente que no existe una oposición fundamental entre los órganos estatales (es decir, los órganos federales y regionales) por un lado y los órganos del autogobierno local por el otro. Las actividades de los órganos locales, sobre todo su competencia, dependen directamente de las decisiones legislativas de la federación y de las regiones.

El establecimiento de un estatus legal diferente de las autoridades públicas sobre una base territorial con la atribución de autoridades de uno u otro nivel territorial con un cierto grado de independencia es característico de la mayoría de los estados actuales.

Sin embargo, no todos los sistemas legales practican la distinción estricta entre la naturaleza jurídica de las autoridades estatales y locales. Entonces, ¿existe en este sentido una diferencia fundamental entre la institución del autogobierno local en Rusia y la institución similar en otros estados? Parece que no es así.

Parece muy probable que la distinción entre las autoridades públicas estatales y locales en Rusia tenga como objetivo enfatizar el estatus legal especial de los órganos locales, teniendo en cuenta la experiencia histórica. Como ya ha quedado dicho, durante el período soviético, que precedió a la adopción de la Constitución de Rusia de 1993, el autogobierno local no existía en su comprensión actual.

La Constitución de Rusia de 1993, para garantizar la existencia del autogobierno local como una institución necesaria en un estado democrático, fijó intencionalmente el estatus especial de las autoridades locales. Eso fue el fundamento para el desarrollo posterior de esta institución a fin de evitar el establecimiento de un sistema jerárquico de las autoridades públicas (cuando los órganos locales de facto tienen muy poca independencia), lo que existió en el período soviético.

Por tanto, coincidimos con José Luis Meilán Gil cuando afirma que "una larga tradición centralizadora no puede acabarse súbitamente por una decisión, aunque ésta sea del alcance de una nueva Constitución"14.

La distinción entre las autoridades públicas estatales y locales, establecida en el artículo 12 de la Constitución de Rusia, adquirió importancia práctica casi inmediatamente después de la adopción de la nueva Constitución. Sobre la base de la norma del artículo 12 de la Constitución de Rusia, el Tribunal Constitucional de la Federación de Rusia en los primeros años después de la adopción de la Constitución de facto defendió la institución del autogobierno local, señalando reiteradamente la imposibilidad de su abolición.

En particular, en la sentencia n. ${ }^{\circ} 15-\mathrm{P}$, del 30 de noviembre de 2000 , el Tribunal Constitucional, en referencia a los artículos 3 y 12 de la Constitución de Rusia, declaró la inconstitucionalidad de las disposiciones del Estatuto de

14 Meilán Gil, J. L. Autonomias y descentralización local. Revista de Estudios de la Administración Local y Autonómica, 1989, n. ${ }^{\circ}$ 243, p. 552. 
la Región de Kursk que "permiten la abolición del autogobierno local como tal en un territorio local por las autoridades estatales, aunque si la decisión de negarse a ejercer el derecho a organizar el autogobierno local sea aprobada de manera independiente y voluntaria a través de un referéndum por la mayoría de la población de este territorio". Según el Tribunal Constitucional de la Federación de Rusia, "tanto el propio territorio local como el derecho de los ciudadanos que viven en el territorio a ejercer el autogobierno local surgen sobre la base de la Constitución de la Federación de Rusia y la ley, pero no sobre la base de la voluntad de la población del territorio local".

El ejemplo anterior no fue el único cuando las autoridades regionales prácticamente intentaron abolir el autogobierno local dentro de los límites de una región bajo varios pretextos. Sin embargo, esta práctica fue bloqueada sistemáticamente por el Tribunal Constitucional de la Federación de Rusia con referencia a las disposiciones de los artículos 3 y 12 de la Constitución de Rusia.

A este respecto se puede constatar que los autores del texto de la Constitución rusa previeron el problema latente y, teniendo en cuenta la experiencia soviética en organizar la autoridad pública (y, como consecuencia, la mentalidad correspondiente de los líderes regionales, que a menudo se quedaron en sus puestos incluso después del cambio del régimen político), enfatizaron en el texto de la Constitución necesidad de la existencia de la institución del autogobierno local y fortalecieron intencionalmente sus garantías.

Entonces, la Constitución de la Federación de Rusia establece un modelo de organización de la autoridad pública en el que establecimiento en el ámbito local de un sistema jerárquico según el principio de "autoridad-obediencia" (de facto, el modelo soviético) no está permitido.

Hasta hace poco parecía que tal modelo de organización de las autoridades públicas era inquebrantable y que los órganos estatales no deberían en ningún caso influir en las actividades de los órganos del autogobierno local, con la excepción de la regulación legislativa de sus actividades y el control del ejercicio de sus competencias en la forma, prescrita por la ley.

Sin embargo, en 2015 se adoptaron enmiendas a la legislación federal con respecto a la formación de los órganos del autogobierno local. Una de las innovaciones fue el procedimiento para elegir al alcalde. Anteriormente, este último pudo ser elegido por la población en las elecciones locales o por un órgano representativo de entre los diputados locales. Pero después de la reforma se hizo posible otra opción para elegir al alcalde: elección por un órgano representativo de entre los candidatos, representados por una comisión competitiva especial, y en algunos territorios locales la mitad de los miembros de dicha comisión son nombrados por el gobernador regional (es decir, por el órgano estatal).

La nueva opción para elegir al alcalde se ha generalizado bastante en las regiones rusas, especialmente donde los candidatos de la oposición podrían ganar las elecciones. 
Hay que tener en cuenta que incluso antes de las enmiendas legislativas correspondientes, los gobernadores de los sujetos de la federación de facto tenían una fuerte influencia en las actividades de los órganos locales. Después de aprobar dichas enmiendas, el procedimiento para elegir al alcalde en algunos territorios locales parece más al procedimiento de nombramiento (por el poder estatal).

La aprobación de estas enmiendas provocó una crítica en la doctrina científica rusa (pero, por supuesto, no por todos los científicos). Los autores señalaron que tales cambios fortalecen la centralización del poder, mientras que el grado de centralización ya es tan alto que amenaza la abolición de la institución del autogobierno local ${ }^{15}$. En la doctrina rusa también se señala que esos cambios no tenían como objetivo tener en cuenta las características geográficas, culturales o históricas de las regiones rusas, sino que tener en cuenta solo una característica: el estado de la política regional y la correlación de las fuerzas políticas en los sujetos de la Federación. Entonces, el autogobierno local depende en gran medida de la situación política regional ${ }^{16}$.

Sin embargo, el Tribunal Constitucional de la Federación de Rusia reconoció la constitucionalidad de las nuevas disposiciones de la ley federal ${ }^{17}$.

Y en 2020 el Estado decidió ir más allá. Se trata de las enmiendas a la Constitución de Rusia aprobadas en julio de 2020. La reforma constitucional contiene decisiones bastante inesperadas que no han sido investigadas suficientemente por la comunidad científica y jurídica en general y que también afectan al autogobierno local. Por primera vez en la historia de la Constitución actual, se ha reformado el capítulo 8, "El autogobierno local".

Como resultado de la reforma constitucional, en el artículo 132 de la Constitución rusa apareció una disposición según la cual "los órganos del autogobierno local y los órganos del poder estatal son parte de un sistema unido de autoridad pública en la Federación de Rusia e interactúan para la resolución más efectiva de los asuntos en interés de la población que vive en el territorio correspondiente".

O sea: si el artículo 12 de la Constitución rusa indica que los órganos del autogobierno local no están incluidos en el sistema de los órganos estatales, el artículo 132 de la nueva edición establece que ambos están incluidos en el sistema unido de autoridades públicas.

15 VASILIEV, V. I. Mestnoe samoupravlenie na puti tsentralizatsii i sokrashcheniya vybornosti [Local Self-Government on the Way to Centralization and Reduction of Electivity]. Journal of Russian Law, 2015, n. ${ }^{\circ}$ 9. Dor: https://doi.org/10.12737/13047 (en ruso).

16 BAZHENOVA, O.I.. K novoy modeli vzaimootnosheniy gosudarstva i mestnogo samoupravleniya.S chego nachat'? [To a new model of relations between the state and local self-government. Where to start?]. "Local Law" Journal, 2018, n. ${ }^{\circ}$ 6, p. 28. Disponible en http://mestnoepravo. com/docs/2018-6-002.pdf (en ruso).

17 Véase la sentencia n. ${ }^{\circ} 30-\mathrm{P}$ del $1 .^{\circ}$ de diciembre de 2015; el auto n. ${ }^{\circ} 663-\mathrm{O}$ del 28 de marzo de 2017; el auto n. ${ }^{\circ} 1568-\mathrm{O}$ del 25 de junio de 2019. 
La enmienda no es indiscutible. Por un lado, el Tribunal Constitucional de la Federación de Rusia ha señalado reiteradamente que tanto los órganos locales como los órganos estatales son autoridades públicas, lo que implica la necesidad de su interacción ${ }^{18}$. Nadie ponía esto en duda, y en este sentido la reforma constitucional no dice nada nuevo.

Por otro lado, la frase "el sistema unido de autoridades públicas" causa discusiones. Como si reescribieran el artículo 12 de la Constitución, y resultó algo que no cumple totalmente con la norma del artículo 12 de la Constitución. No obstante, el artículo 12 de la Constitución tiene prioridad, porque, a diferencia del artículo 132, se encuentra en el capítulo I, y el artículo 132 no puede contradecir el artículo 12.

Otra enmienda sumamente importante es la nueva disposición del artículo 131 de la Constitución de Rusia, según la cual los órganos del poder estatal pueden participar en la formación de los órganos del autogobierno local, en el nombramiento y la destitución de los funcionarios locales (incluso de los alcaldes) en la forma y en los casos establecidos por una ley federal.

Dichas enmiendas confirman la tendencia de los cambios legislativos en los últimos diez años que muestra el camino hacia la centralización y la reducción de la independencia de los órganos locales. Pero si antes la posibilidad de participar en la formación de los órganos del autogobierno local por parte de los órganos estatales fue establecida solo en la legislación ordinaria, ahora esta posibilidad ya es adoptada en el ámbito constitucional.

No hay que olvidar que la naturaleza del autogobierno local en Rusia aún no está completamente aclarada, porque, como se señala en la doctrina, el autogobierno local es un tipo especial de poder, que contiene algunos atributos del poder estatal, y, simultáneamente, unos atributos propios ${ }^{19}$. Por lo tanto, y como es obvio, de la regulación normativa depende mucho el desarrollo futuro de la naturaleza del autogobierno local, si este va a ir hacia la centralización o la descentralización, hacia lo estatal o hacia lo comunal.

La reforma constitucional parece ser una señal obvia y significativa para un mayor desarrollo de la legislación sobre el autogobierno local. Es muy probable que este desarrollo siga el camino de una mayor centralización del poder a través de las enmiendas a la legislación ordinaria sobre el autogobierno local (sobre la base de las normas constitucionales reformadas). Las competencias de los órganos estatales para la formación de los órganos del

18 Véase, por ejemplo, la sentencia del Tribunal Constitucional de la Federación de Rusia n. ${ }^{\circ}$ 1-P del 24 de enero de 1997; la sentencia del Tribunal Constitucional de la Federación de Rusia n. ${ }^{\circ}$ 3-P del 15 de enero de 1998.

19 Peshin, N. L. Munitsipal'naya vlast': prodolzhenie gosudarstva ili institut samoorganizatsii obshchestva? [Municipal Power: Continuation of the State Power or Institution of SelfOrganization of Society? ]. Proceedings of VSU. Series: Law, 2019, n. ${ }^{\circ} 2(37)$, p. 43. Disponible en: http://www.vestnik.vsu.ru/pdf/pravo/2019/02/2019-02-07.pdf (en ruso). 
autogobierno local pueden ampliarse y la participación ciudadana en ese proceso a través de las elecciones puede reducirse.

Tal escenario (si se adoptan las enmiendas correspondientes a la legislación ordinaria) tendrá una serie de consecuencias negativas, incluso para el desarrollo de la sociedad civil en Rusia.

El proceso de desarrollo de la sociedad civil durante el período soviético tenía ciertas dificultades por varias razones. Como señala Abrámova M., la vigente Constitución rusa "abandona la tradición soviética previa, en la cual existía la supremacía de la sociedad sobre el individuo. Por lo tanto, en la URSS existía la supremacía del sistema de derechos económicos y sociales sobre los derechos personales. La nueva Constitución rusa hace hincapié en los derechos políticos" ${ }^{20}$.

En las nuevas condiciones se suponía que el autogobierno local se convertiría en uno de los factores para el desarrollo de la sociedad civil, ya que en teoría es en el ámbito local donde se resuelven los problemas de la vida cotidiana de los ciudadanos, y los órganos del autogobierno local son los más cercanos a la población. Como señala A. A. Uvárov, "el autogobierno local es el puente entre la sociedad civil y el Estado"21.

Sin embargo, N. S. Timoféev afirma que "en la actualidad la realidad muestra la violación del principio fundamental del autogobierno: la participación de la población en el sistema de toma de decisiones y en el propio proceso de gestión" ${ }^{22}$. Y eso frena el desarrollo tanto de la sociedad civil como del instituto del autogobierno local.

Además, la fuerte centralización del poder puede provocar una crisis de representación debido a un aumento de la distancia entre la población y los órganos del autogobierno local. Algunas señales de la crisis de representación ya se ven en ciertas regiones de Rusia, las cuales muestran resultados de las elecciones locales y regionales bastante inesperados (Región de Jabárovsk, Región de Vladímir, Región de Novosibirsk).

La falta del diálogo (también a través de las elecciones) entre la población y los órganos del autogobierno local puede llevar a decisiones impopulares entre la población, lo cual puede provocar una crisis política no solo en el ámbito local, sino también en los ámbitos regional y federal.

20 ABrámova, M. Los derechos humanos en el sistema juridico de Rusia: tradición y modernización (análisis comparativo). Boletín Mexicano de Derecho Comparado. Nueva serie, $\mathrm{XLX}, \mathrm{n}$. 149, mayo-agosto de 2017, p. 546. Dor: http://dx.doi.org/10.22201/iij.24484873e.2017.149.11349.

21 Uvárov, A. A. Mestnoe samoupravlenie i grazhdanskoe obshchestvo [Local SelfGovernment and Civil Society]. Constitutional and Municipal law, 2008, n. ${ }^{\circ} 15$. Disponible en: http://lawinfo.ru/catalog/contents-2008/konstitucionnoe-i-municipalnoe-pravo/15/ (en ruso).

22 Timofeev, N. S. Problemy uchastiya mestnogo samoupravleniya i grazhdanskogo obshchestva $v$ sisteme rossiyskogo etatizma [Problems of Participation of Local Government and Civil Society in the System of Russian Etatism]. Constitutional and Municipal law, 2015, n. ${ }^{\circ} 10$, pp. 12-17. Disponible en: http://lawinfo.ru/catalog/contents-2015/konstitucionnoe-i-municipalnoepravo/10/ (en ruso). 
Las autoridades estatales obviamente no están interesadas en tales consecuencias. En este sentido, para el desarrollo del autogobierno local, de la sociedad civil y del diálogo entre la sociedad civil y el poder estatal se debe frenar la tendencia hacia la centralización del poder. Coincidimos con L. T. Chikhladze y A. A. Lárichev cuando afirman que "la intervención estatal implica la implementación del concepto de 'centralización razonable' -fortalecer el papel del Estado en la implementación de los fundamentos organizativos y funcionales del autogobierno local en casos estrictamente limitados por una ley, sin violar las disposiciones del artículo 12 de la Constitución de Rusia" ${ }^{23}$.

\section{El derecho a ejercer el autogobierno local}

Sobre la base de la interpretación sistemática de las disposiciones de la Constitución de Rusia, el Tribunal Constitucional de la Federación de Rusia concluyó que la Constitución también establece "el derecho a ejercer el autogobierno local".

A pesar de que el capítulo 2 de la Constitución rusa, "Los derechos y libertades del ser humano y del ciudadano", no establece directamente el derecho a ejercer el autogobierno local, el Tribunal Constitucional de la Federación de Rusia señala que tal derecho existe.

En la sentencia n. ${ }^{\circ}$ 7-P del 2 de abril de 2002 el Tribunal Constitucional señaló que "[...] no se excluye la protección mediante la justicia constitucional de los derechos de las entidades locales como asociaciones territoriales de los ciudadanos que colectivamente ejercen el derecho al autogobierno local sobre la base de la Constitución de la Federación de Rusia [...]”.

En el auto n. $.^{\circ} 2002-O$ del 29 de septiembre de 2015 el Tribunal Constitucional señaló que "[...] no puede ser desconocida la especificidad del derecho constitucional al autogobierno local, que, al garantizar la participación de los ciudadanos en resolución independiente por parte de la población de los asuntos de importancia local, se caracteriza por la unidad de los fuentes individuales y colectivos [...]".

Numerosas son otras decisiones en las que el Tribunal Constitucional reconoció la existencia del derecho al autogobierno local ${ }^{24}$.

Parece que no hay razones políticas y legales para no estar de acuerdo con el Tribunal Constitucional de la Federación de Rusia en la medida en

23 Chikhladze, L. T., Larichev, A. A. (2020) The dualistic model and "rational centralization" as factors of the effective functioning of local government within the Russian public authority mechanism. RUDN Journal of Law. 24 (2), pp. 233-251. Dor: 10.22363/2313-2337-202024-2-233-251.

24 Véase, por ejemplo, la sentencia del Tribunal Constitucional de la Federación de Rusia n. ${ }^{\circ}$ 1-P del 24 de enero de 1997; el auto del Tribunal Constitucional de la Federación de Rusia n. ${ }^{\circ}$ 171-O-P del 3 de abril de 2007; la sentencia del Tribunal Constitucional de la Federación de Rusia n. ${ }^{\circ}$ 26-P del 3 de julio de 2019. 
que el derecho a ejercer el autogobierno local pueda ser reconocido y tener la protección adecuada.

Esta conclusión se basa en las normas sobre el autogobierno local (del capítulo 1 y del capítulo 8 de la Constitución de Rusia), y también en las normas del capítulo 2 de la Constitución, que, aunque no establecen directamente dicho derecho, sino que lo confirman indirectamente en algunas normas (en particular, los párrafos 1 y 2 del artículo 32 de la Constitución).

No hay que olvidar sobre el párrafo 1 del artículo 55 de la Constitución de Rusia, según el cual "la enumeración de los principales derechos y libertades en la Constitución de la Federación de Rusia no se debe interpretar como renuncia o menosprecio de otros derechos y libertades del ser humano y del ciudadano, generalmente reconocidos".

Según el artículo 133 de la Constitución de Rusia el autogobierno local tiene garantizado el derecho a la defensa judicial, pero no hay una especificación de esta disposición en el texto de la Constitución de Rusia.

Según el párrafo 4 del artículo 125 de la Constitución de la Federación de Rusia, el Tribunal Constitucional de la Federación de Rusia, por queja sobre la violación de los derechos y libertades constitucionales de los ciudadanos y a solicitud de los tribunales, verificará la constitucionalidad de una ley aplicada o que será aplicada en un caso concreto, de conformidad con el procedimiento establecido por la ley federal.

En este sentido, el reconocimiento del autogobierno local como un derecho inalienable de los ciudadanos y sus asociaciones es de gran importancia legal, ya que sobre la base de dicho reconocimiento el Tribunal Constitucional de la Federación de Rusia concluyó que los órganos locales y los ciudadanos pueden interponer recursos de inconstitucionalidad de las leyes en el Tribunal Constitucional cuando tales leyes violaren el derecho al autogobierno local ${ }^{25}$. Es curioso que unos años antes el mismo Tribunal Constitucional concluyera que los órganos locales no tienen acceso a la justicia constitucional ${ }^{26}$, pero esta posición ya es parte de la historia y hace muchos años perdió su actualidad.

Por todo ello, el acceso a la justicia constitucional para los órganos locales y para los ciudadanos (sobre nuestra materia) no está directamente establecido en la ley, sino que el Tribunal Constitucional de la Federación de Rusia lo reconoce sobre la base de una intencionada interpretación amplia de las normas de la Constitución de Rusia.

Dicha posición del Tribunal Constitucional se ve razonable, pero al mismo tiempo hay que reconocer un vacío legal correspondiente. Resulta que está reconocido el derecho de los órganos locales de interponer recursos de

25 La sentencia del Tribunal Constitucional de la Federación de Rusia n. ${ }^{\circ}$ 7-P del 2 de abril de 2002.

26 El auto del Tribunal Constitucional de la Federación de Rusia n. ${ }^{\circ} 20-\mathrm{O}$ del 19 de marzo de 1997. 
inconstitucionalidad, pero el procedimiento para la implementación de dicho derecho no ha sido establecido por una ley. Por lo tanto, no se tienen en cuenta los detalles del estatus legal de los órganos locales (o de la población de un territorio local). Algunos aspectos de dicho procedimiento también se desarrollan exclusivamente por la práctica del Tribunal Constitucional.

Además, en la doctrina rusa se señala el problema de distinguir entre el derecho de un ciudadano por un lado y el derecho de un territorio local y sus órganos por otro lado para interponer recursos de inconstitucionalidad. La profesora de la Universidad Estatal de San Petersburgo Gritsenko E. V. y el profesor de la Universidad de Humboldt de Berlín Rosemary W. dicen que la legislación rusa y la práctica judicial no establecen claramente quién tiene el derecho correspondiente, si son los ciudadanos o los territorios locales (representados por los órganos correspondientes). Eso plantea ciertos problemas que influyen negativamente en las garantías constitucionales del autogobierno local ${ }^{27}$.

Cabe señalar que los sistemas jurídicos de otros países (por ejemplo, de Alemania o España) conocen los tipos especiales de recursos judiciales sobre violaciones de los derechos del autogobierno local, que se recurren de acuerdo con un procedimiento especialmente establecido para esto y tienen en cuenta los detalles correspondientes. La Federación de Rusia debería analizar la experiencia relevante de los países extranjeros y considerar la posibilidad de su implementación en la legislación nacional, teniendo en cuenta todas las ventajas y desventajas de esos procedimientos. Esto simplificará y hará más comprensible y predecible el procedimiento para defender el derecho al autogobierno local.

III. EL CAPÍTULO 8, "EL AUTOGOBIERNO LOCAL",

DE LA CONSTITUCIÓN DE RUSIA

Además de las normas anteriores, que establecen los principios básicos de organización de la autoridad pública en el ámbito local y son de particular importancia, ya que se encuentran en los capítulos 1 y 2 de la Constitución de Rusia, la Constitución de Rusia también contiene un capítulo particular sobre el autogobierno local.

El capítulo 8 de la Constitución de Rusia, que consta de los artículos 130-133, contiene ciertas disposiciones organizativas y funcionales del autogobierno local. A diferencia de las normas de los artículos 3 o 12, que por

27 GricenKo, E. V., Rosemary, W. Zashchita prav mestnogo samoupravleniya sredstvami konstitutsionnogo pravosudiya $v$ Rossii i Germanii [Local self-government rights protection by means of constitutional justice in Russia and Germany]. Journal of Constitutional Justice, 2015. n. ${ }^{\circ}$ 4. pp. 22-38 (en ruso). Disponible en: http://lawinfo.ru/catalog/contents-2015/zhurnalkonstitucionnogo-pravosudija/4/ 
su naturaleza son normas-principios, las normas de los artículos 130-133 de la Constitución de Rusia son en su mayoría las normas más concretas.

El artículo 130 de la Constitución de Rusia consagra la naturaleza del autogobierno local, indicando que esto "garantiza a la población la solución de los problemas de importancia local, el dominio, el uso y la disposición de la propiedad municipal (local)". Este artículo además indica que el autogobierno local lo ejercen los ciudadanos mediante los referéndums, las elecciones y otras formas de expresión de la libre voluntad, directa o a través de los órganos (electivos y otros) del autogobierno local. En esencia esta norma repite en gran medida la norma del artículo 3 de la Constitución de Rusia, pero la desarrolla en cierto grado.

El artículo 131 de la Constitución de Rusia está dedicado a la organización territorial del autogobierno local y antes de la adopción de las enmiendas a la Constitución establecía que:

- el autogobierno local se ejerce en unas localidades (poselenie en ruso) urbanas y rurales $y$ en otros territorios habida cuenta de las tradiciones históricas y otras tradiciones locales;

- la estructura de los órganos del autogobierno local se determina por la población en forma independiente;

- la modificación de las fronteras de los territorios en los cuales se ejerce el autogobierno local se puede realizar sólo teniendo en cuenta la opinión de la población de los correspondientes territorios.

Hasta hace poco las localidades urbanas y rurales eran, como regla general, los territorios locales básicos (generalmente son bastante pequeños en territorio y población) en un sistema de los territorios locales de dos niveles. Una comarca (municipal'niy raión, en ruso) agrupa varias localidades urbanas y rurales y es el territorio local de segundo nivel. Para localidades es obligatorio formar parte de una comarca. O sea, si el autogobierno local se ejerce en las localidades, siempre se trata de los territorios locales de dos niveles: los más pequeños y más cercanos a la población (localidades) y los más grandes (comarcas).

Como la excepción a esta regla la ley reconoce unas ciudades relativamente grandes, que son territorios locales independientes -distritos urbanos (gorodskóy ókrug, en ruso)- y no forman parte de las comarcas. Entonces, si el autogobierno se ejerce en los distritos urbanos, se trata de un territorio local de un solo nivel (salvo que estos distritos urbanos se dividan a los territorios locales más pequeños [los barrios], pero en la práctica no es frecuente que ese modelo suela utilizarse).

Además, una serie de especialidades (incluso desde el punto de vista de organización territorial) se han establecido para las ciudades de importancia federal que son no solo las ciudades sino también los sujetos de la federación. 
Hasta el año 2019 así era el sistema de los territorios en los cuales se ejerce el autogobierno local, que implicaba los territorios como localidades urbanas y rurales, comarcas, distritos urbanos y los territorios locales en las ciudades de importancia federal.

Sin embargo, en 2019 se llevó a cabo otra reforma de la legislación sobre el autogobierno local ${ }^{28}$. Como el resultado de la reforma, a la legislación federal se ha adicionado un nuevo tipo de territorio local: un distrito municipal (municipal'niy ókrug, en ruso). El distrito municipal es un territorio local de un solo nivel (o sea: no forma parte de una comarca ni de otro territorio local) y consiste en asentamientos rurales.

La nueva ley aclara los requisitos que los distritos urbanos deben cumplir (en particular, en términos de densidad de población y de porcentaje de ciudadanos que viven en ciudades y no en asentamientos rurales). Los distritos urbanos que no cumplan con los nuevos requisitos deben transformarse al distrito municipal hasta 2025.

Además, y eso es lo más importante, la nueva ley permite la transformación de las comarcas en distritos municipales. En este caso las comarcas, así como las localidades rurales y urbanas que forman parte de tal comarca, pierden el estatus de un territorio donde se ejerce el autogobierno local. Es decir, en este caso en lugar de dos niveles de territorios locales existirá un territorio local (con tamaño de una comarca) de un solo nivel.

En el caso de la aplicación amplia de las nuevas reglas de organización territorial del autogobierno local en las regiones rusas, esto llevará a que el autogobierno local se ejerce principalmente en los territorios locales bastante grandes de un solo nivel, cuyos órganos, de acuerdo con la práctica e incluso con la legislación rusa, interactúan más estrechamente con las autoridades estatales y están más lejos de la población local.

Las enmiendas a la Constitución debilitaron aún más el estatus de las localidades rurales y urbanas. La nueva edición del artículo 131 de la Constitución establece que el autogobierno local se ejerce en territorios locales los tipos de los cuales son establecidos por una ley federal. O sea, la Constitución de Rusia ya no menciona que el autogobierno local se ejerce en las localidades rurales y urbanas. En este sentido, las enmiendas a la Constitución de Rusia corresponden a la tendencia del desarrollo de la legislación sobre el autogobierno local en los últimos años.

Entonces, si antes las localidades rurales y urbanas eran generalmente los territorios locales básicos, ahora están por perder su importancia. Esto puede traer un distanciamiento de los órganos locales de la población, lo cual no favorece a la implementación del principio de subsidiariedad, establecido en la Carta Europea de la Autonomía Local. 
Además, el artículo 131 de la Constitución rusa se complementó con una disposición según la cual las características del ejercicio del poder público (no del autogobierno local) en los territorios de las ciudades de importancia federal, los centros administrativos (capitales) de los sujetos de la Federación de Rusia y en otros territorios pueden establecerse por ley federal. El significado de esta norma aún no ha sido aclarado, pero es probable que en las ciudades más grandes la influencia de los órganos estatales se fortalezca aún más.

El artículo 132 de la Constitución de Rusia establece las competencias básicas de los órganos locales:

- administración de propiedad de los entes locales;

- formación, aprobación y ejecución del presupuesto local;

- establecimiento de impuestos y tasas locales;

- efectuación de la protección del orden público;

- resolución de otros problemas de importancia local.

Las competencias particulares de los órganos locales se establecen por la legislación ordinaria (principalmente federal).

Cabe señalar que el artículo 132 de la Constitución de Rusia se complementó con una disposición según la cual los órganos del autogobierno local garantizan, dentro de su competencia, la accesibilidad de la atención médica. En la versión inicial del proyecto de ley sobre enmiendas a la Constitución no existía tal disposición. Esa disposición apareció durante el agravamiento de la situación con la pandemia de coronavirus, lo cual indica que el legislador ha respondido rápidamente a los desafíos actuales para el sistema sanitario nacional.

Además, el artículo 132 de la Constitución de Rusia establece que los órganos estatales pueden delegar en los órganos del autogobierno local competencias estatales con asignación de medios materiales y financieros necesarios.

La legislación ordinaria va más allá y establece que las competencias de los órganos locales pueden ser redistribuidos por los órganos regionales a favor de los últimos, si esto está previsto en las leyes federales. En la práctica, este mecanismo se usa con bastante frecuencia, así como el mecanismo de la delegación. Teniendo en cuenta que muchas de las competencias de los órganos locales son delegadas (y, por lo tanto, se ejercen bajo el control de las autoridades estatales) y que además las competencias de los órganos locales se pueden redistribuir, a menudo no se ve muy claro la "frontera" entre los órganos locales y los órganos estatales. Algunos autores, al analizar los procesos de la delegación y la redistribución de las competencias en el ámbito local en Rusia, señalan que dichos mecanismos influyen bastante en 
el proceso de la transformación real del autogobierno local en uno de los niveles del poder ejecutivo ${ }^{29}$.

El artículo 133 de la Constitución de Rusia determina algunas garantías del autogobierno local:

- el derecho a la defensa judicial;

- el derecho a una compensación de gastos adicionales;

- la prohibición de limitar los derechos del autogobierno local establecidos por la Constitución de Rusia y las leyes federales.

Como resultado de la reforma constitucional, la norma del artículo 133 de la Constitución ha sido reformada. En lugar de "derecho a una compensación de gastos adicionales derivados de las decisiones, tomadas por los órganos estatales", la nueva versión del artículo 133 de la Constitución establece "el derecho a una compensación de gastos adicionales derivados del cumplimiento por parte de los órganos de autogobierno local, en cooperación con los órganos del poder estatal, de funciones públicas".

Entonces, si antes la base de la compensación eran las decisiones de los órganos estatales, en la nueva versión de la norma constitucional la base de la compensación es la solución conjunta de ciertos asuntos de importancia estatal por los órganos locales y estatales.

Esto reduce las condiciones para que los órganos locales compensen los gastos adicionales y, por lo tanto, reduce las garantías de los propios órganos locales a favor de los órganos estatales.

\section{CONCLUSIONES}

La regulación constitucional del autogobierno local en la Federación de Rusia se ve bastante extensa. En esto la experiencia rusa es más colombiana, por ejemplo, que europea. La Constitución de Rusia contiene bastantes normas sobre el autogobierno local. Las normas constitucionales sobre el autogobierno local tienen diferente naturaleza jurídica y significado.

Las normas del capítulo 1, "Los fundamentos del sistema constitucional", de la Constitución de Rusia son las normas-principios y establecen las disposiciones básicas sobre las que se basa el sistema de autoridad pública en la Federación de Rusia. La norma principal del primer capítulo de la Constitución de Rusia es la norma del artículo 12, que reconoce el autogobierno local como uno de los fundamentos del sistema constitucional del país y

29 Zubarev, S. M. K voprosu ob ogosudarstvlenii mestnogo samoupravleniya [The issue of governmentalization of local self-government]. Lex russica, 2018, n. ${ }^{\circ} 12$ (145), p. 88. DoI: 10.17803/1729-5920.2018.145.12.083-089 (en ruso). 
establece la distinción entre las autoridades públicas en Rusia en las estatales (federales y regionales) y las locales.

Un análisis completo de las normas del capítulo 2 de la Constitución de Rusia permitió al Tribunal Constitucional de la Federación de Rusia reconocer la existencia del derecho al autogobierno local entre los derechos y libertades consagrados en Rusia. El principal efecto de tal interpretación fue el reconocimiento del derecho a interponer recursos de inconstitucionalidad por parte de los órganos locales y de los ciudadanos.

Las normas del capítulo 8 de la Constitución de Rusia establecen disposiciones sobre el procedimiento para la implementación del autogobierno local, la organización territorial del autogobierno local, algunas competencias y garantías del autogobierno local.

Cabe señalar que una regulación tan detallada del autogobierno local en el texto de la Constitución no es una práctica común. En muchos países la Constitución se limita a pocos artículos sobre el autogobierno local. En este caso el contenido de las normas constitucionales se aclara en la legislación ordinaria y en la jurisprudencia del Tribunal Constitucional sobre la base de los principios generales, establecidos en la Constitución.

La regulación constitucional del autogobierno local en Rusia en este sentido se destaca incluso en el contexto de las repúblicas exsoviéticas, que también suelen reglamentar en detalles ciertas cuestiones en los textos constitucionales.

Parece que este enfoque se ha desarrollado en relación con las características históricas del desarrollo de la legislación rusa. El concepto positivista en el período soviético, que consideraba el derecho solo como "lo que está escrito en la ley", no podía sino afectar el estilo de la regulación jurídica actual. Cabe mencionar que la regulación del autogobierno local en la Constitución de Rusia hizo posible guardar esta institución en los primeros años después de la adopción de la Constitución.

Al mismo tiempo, hay que reconocer que la práctica legislativa y judicial actual ha demostrado que incluso una regulación constitucional detallada no garantiza el desarrollo rápido y efectivo del principio de descentralización del poder en el ámbito local. Las normas de la Constitución de Rusia a menudo se interpretan de manera excesivamente amplia, a causa de que el legislador federal tiene amplias capacidades para la regulación jurídica del autogobierno local. Esta práctica es reconocida por el Tribunal Constitucional de la Federación de Rusia.

Como un ejemplo, a pesar de que el artículo 12 de la Constitución de Rusia establece que los órganos locales no están incluidos en el sistema de los órganos estatales, según la ley federal los órganos estatales en algunos casos participan en el procedimiento de la formación de los órganos locales. La reforma constitucional de 2020 reconoció esta práctica en el ámbito constitucional.

La norma del artículo 131 de la Constitución de Rusia (que indica que la estructura de los órganos locales se determina por la población de forma 
independiente), por el contrario, se interpreta por el legislador y el Tribunal Constitucional de manera restrictiva. La legislación federal y parcialmente la regional establecen la estructura y los tipos de los órganos locales, el procedimiento para su formación y disolución. En este sentido, la población prácticamente no tiene posibilidades de una verdadera determinación independiente de la estructura de los órganos del autogobierno local. Para ser justos, hay que señalar que la redacción de la norma del artículo 131 de la Constitución de Rusia no parece perfecta, ya que es evidente que la población no puede determinar la estructura de los órganos locales completamente independientemente. Sin embargo, la práctica legislativa indica que la población prácticamente no participa en la determinación de la estructura de los órganos locales o al menos del procedimiento para su formación.

Incluso aquellas enmiendas a la legislación ordinaria que, según el legislador, estaban dedicadas a fortalecer el papel de los diputados locales, por ejemplo, la introducción de un "filtro municipal" (el procedimiento para recoger firmas de diputados locales en apoyo de los candidatos en las elecciones regionales, establecido por la legislación rusa), a menudo simplemente limitan la competencia política ${ }^{30}$.

También existe un predominio significativo de la regulación federal sobre el autogobierno local, que generalmente no es típico para los estados federales. Sin embargo, este problema no tiene una solución sencilla. En este sentido llama la atención el punto de vista de O. I. Bazhénova, quien señala que la cuestión de las competencias de los sujetos de la Federación es bastante complicada, porque "durante el período de vigencia de la Ley Federal n. ${ }^{\circ} 154-\mathrm{FZ}$ los sujetos de la Federación a menudo utilizaron sus poderes discrecionales en contra de la orientación, declarada por el estado, de apoyar la reactivación del autogobierno local. Ahora sus acciones encaminadas a la supresión total del autogobierno local no encuentran resistencia por parte de la Federación. En otras palabras, la cuestión de las competencias de los sujetos de la Federación en el ámbito de la organización del autogobierno local es generalmente una cuestión de política estatal"31.

Por todo ello, la amplia regulación constitucional del autogobierno local no garantiza su rápido desarrollo en el camino hacia la descentralización del poder en el ámbito local. La legislación ordinaria y la jurisprudencia del Tribunal Constitucional tienen mucha importancia en este proceso. Precisamente

30 ChIKHLADZE L.T. y GoRYAChIH,A.A. K voprosu o tselesoobraznosti sohraneniya instituta «munitsipal'nogo fil'tra» $v$ Rossiyskoj Federatsii [On the appropriateness of maintaining the institute "municipal filter" in the Russian Federation]. Education and law, 2018, n. ${ }^{\circ}$ 2, p. 44 (en ruso). Disponible en http://education.law-books.ru/?p=1306.

31 BAZENOVA, O. I. Munitsipal'niy okrug: novoe reshenie staroy problemy ili novaya problema organizatsii mestnogo samoupravleniya $v$ Rossii? [Municipal district: a new solution of the old problem or a new problem of local self-government arrangement in Russia?]. Constitutional and Municipal law, 2020 , n. ${ }^{\circ}$. Dor: 10.18572/1812-3767-2020-1-58-66 (en ruso). 
el Tribunal Constitucional puede limitar al legislador si este último interpreta las normas de la constitución de manera demasiado amplia, no a favor del autogobierno local. Sin embargo, en este contexto mucho depende de la situación política en cierto período y del grado de independencia del mismo Tribunal Constitucional. En este sentido coincidimos plenamente con Francisco Javier Díaz Revorio cuando afirma que "[...] tampoco puede haber democracia si no hay una garantía de los propios procesos democráticos por parte de una justicia independiente [...]. Como es evidente, dada la especial función de la justicia constitucional, si esta no es independiente peligra directamente el entero edificio constitucional [... $]^{\prime 32}$. Deberíamos añadir que lo mismo se puede afirmar no solo sobre el edificio constitucional, sino también sobre el desarrollo del autogobierno local.

Teniendo en cuenta que la institución del autogobierno local en Rusia ha recibido su lugar y protección garantizados en el marco del sistema de autoridad pública en Rusia, parece conveniente tomar medidas adicionales para fortalecer la descentralización del poder en el ámbito local. Parece necesaria una redistribución de los recursos financieros en favor de las entidades territoriales locales con la preservación y, en algunos casos, el aumento de las competencias de los órganos locales.

Recordando artículo 12 de la Constitución de Rusia, es necesario reducir la posibilidad de una participación incluso indirecta de las autoridades estatales en la formación de los órganos locales y establecer la prevalencia absoluta de los mecanismos electivos en este asunto.

Desde el punto de vista de organización territorial del autogobierno local, el legislador federal y regional debería tomar medidas para garantizar que relativamente pequeños localidades urbanos y rurales sigan siendo las entidades territoriales local básicas, cuyos órganos son más cercanos a la población local. Y si no lo hacen, entonces el Tribunal Constitucional debería interpretar de manera más restrictiva las disposiciones de la Constitución y, en cierta medida, limitar las competencias del legislador.

Pero, sin embargo, los cambios legislativos recientes (sobre todo la reforma constitucional de 2020) indican una aproximación entre los órganos locales y estatales y un aumento en la influencia de estas últimas, en lugar de una descentralización del poder en el ámbito local y un aumento en la autonomía de los órganos locales. La impresión es que hay un pequeño giro hacia el modelo soviético cuando el Estado no se atreve a "dejar ir" a los órganos locales en una "navegación" relativamente libre.

Pero todavía hay razones para creer que la regulación constitucional actual no permitirá un retorno completo al modelo soviético de organización de la

32 Díaz Revorio, F. J. Cuando el garante de la constitución se convierte en su mayor enemigo. Revista Española de Derecho Constitucional, 2018, n. ${ }^{\circ}$ 113, pp. 348-349. Disponible en www.cepc.gob.es/publicaciones/revistas/revistaselectronicas?IDR=6\&IDN=1397\&IDA=38450. 
autoridad pública en el ámbito local, y las últimas tendencias legislativas son solo una de las etapas en el camino hacia el desarrollo de la institución del autogobierno local.

Entre estas razones se encuentran el reconocimiento del autogobierno local como el fundamento del sistema constitucional y la imposibilidad de reformar el capítulo 1 de la Constitución de Rusia (a menos que se adopte una nueva Constitución, para lo cual actualmente no hay requisitos previos jurídicos ni políticos); y también el reconocimiento por parte del Tribunal Constitucional del derecho al autogobierno local, por lo que los órganos locales y la población local tienen acceso a la justicia constitucional.

En la doctrina se señala que la legislación sobre el autogobierno local en Rusia se desarrolla cíclicamente, con la alternancia de centralización y descentralización ${ }^{33}$. En consecuencia, la siguiente etapa en el desarrollo del autogobierno local en Rusia debería ser la descentralización del poder público.

\section{BIBLIOGRAFÍA}

Abrámova, M. Los derechos humanos en el sistema juridico de Rusia: tradición y modernización (análisis comparativo). Boletín Mexicano de Derecho Comparado. Nueva serie, año XLX, n. ${ }^{\circ}$ 149, mayo-agosto de 2017. Dor: http://dx.doi.org/10.22201/iij.24484 873e.2017.149.11349.

Avakyan, S. A. Modernization of public and political relationships and constitutional reformation: issues and prospects. Constitutional and Municipal law, 2019, n. ${ }^{\circ} 9$.

BAZHENOVA, O. I. To a new model of relations between the state and local self-government. Where to start? "Local Law" Journal, 2018, n. 6.

Bazenova, O. I. Municipal district: a new solution of the old problem or a new problem of local self-government arrangement in Russia? Constitutional and Municipal law, 2020, n. ${ }^{\circ}$ 1. DOI: 10.18572/1812-3767-2020-1-58-66.

Chikhladze, L. T., Goryachin, A. A. On the appropriateness of maintaining the institute "municipal filter" in the Russian Federation. Education and law, 2018, n. 2.

Chikhladze, L. T., Larichev, A. A. (2020) The dualistic model and "rational centralization" as factors of the effective functioning of local government within the Russian public authority mechanism. RUDN Journal of Law. 24 (2), pp. 233-251. Dor: 10.22363/23132337-2020-24-2-233-251.

DíAz Revorio, F. J. Cuando el garante de la constitución se convierte en su mayor enemigo. Revista Española de Derecho Constitucional, 2018, n. ${ }^{\circ} 113$.

33 SCHEPACHEV V.A. Respublikanskiy put'razvitiya Rossii nevozmozhen bez sil'nogo mestnogo samoupravleniya [Further republican development of Russia is impossible without strong self-government]. State Power and Local Self-Government, 2018, n. ${ }^{\circ}$ 4, pp. 46-52. Disponible en: http://lawinfo.ru/catalog/contents-2018/gosudarstvennaja-vlast-i-mestnoe-samoupravlenie/4/ (en ruso). 
EREMYAn, V.V. The theoretical grounds of the local's and regional's government reform in the second half of the XLXth century in Russia. RUDN Journal of Law, 2000, $\mathrm{n}{ }^{\circ} 1$.

Gricenko, E. V., Rosemary, W. Local self-government rights protection by means of constitutional justice in Russia and Germany. Journal of Constitutional Justice, 2015. n. ${ }^{\circ}$.

Ignatyuk, N. A., Pavlushrin, A. V. Municipal law. Study guide. Moscow, Yustitsinform, 2007.

Meilán Gil, J. L. Autonomías y descentralización local. Revista de Estudios de la Administración Local y Autonómica, 1989, n. 243.

Peshin, N. L. Municipal Power: Continuation of the State Power or Institution of SelfOrganization of Society? Proceedings of vsu. Series: Law, 2019, n. 2 (37).

Postovor, N. V. Local Self-government. History, theory, practice. Moscow, Advertising and publishing center "Fedorov", 1995.

Schepachev V. A. Further republican development of Russia is impossible without strong self-government. State Power and Local Self-Government, 2018, n. 4.

Shugrina, E. S. The Concept of Local Self-government as a Constitutional Value. Juridical World, 2016, n. ${ }^{\circ}$.

Timofeev, N. S. Problems of Participation of Local Government and Civil Society in the System of Russian Etatism. Constitutional and Municipal law, 2015, n. 10.

Uvarov A. A. Local Self-Government and Civil Society. Constitutional and Municipal law, $2008,{ }^{\circ} 15$.

Vasiliev, V. I. Municipal law of Russia. Textbook. Second edition. Moscow, Yustitsinform, 2012 .

VAsiliev, V. I. Local Self-Government on the Way to Centralization and Reduction of Electivity. Journal of Russian Law, 2015, n. ${ }^{\circ}$ 9. Dor: https://doi.org/10.12737/13047 (en ruso).

Zorkin, V. D. Commentary on the Constitution of the Russian Federation. Second edition (revised). Edited by V. D. Zorkin. Moscow, Norma, 2011.

Zubarev, S. M. The issue of governmentalization of local self-government. Lex russica, 2018, n. ${ }^{\circ} 12$ (145). DOI: 10.17803/1729-5920.2018.145.12.083-089.

\section{Legislación}

Constitución de la Federación de Rusia.

Ley Federal de 28 de agosto de 1995 n. 154-FZ "Sobre los principios generales de organización del autogobierno local en la Federación de Rusia".

Ley Federal de 06.10.2003 n. 131-FZ “Sobre los principios generales de organización del autogobierno local en la Federación de Rusia".

Rusia. Ley Federal del 1. ${ }^{\circ}$ de mayo de 2019 n. $87-F Z$ "Sobre las enmiendas a la Ley Federal 'Sobre los principios generales de organización del autogobierno local en la Federación de Rusia"”. 


\section{Jurisprudencia}

Rusia. Tribunal Constitucional. Sentencia n. ${ }^{\circ}$ 1-P del 24 de enero de 1997.

Rusia. Tribunal Constitucional. Sentencia n. ${ }^{\circ}$ 3-P del 15 de enero de 1998.

Rusia. Tribunal Constitucional. Sentencia n. ${ }^{\circ}$ 15-P del 30 de noviembre de 2000.

Rusia. Tribunal Constitucional. Sentencia n. ${ }^{\circ}$ 7-P del 2 de abril de 2002.

Rusia. Tribunal Constitucional. Sentencia n. ${ }^{\circ}$ 30-P del 1. ${ }^{\circ}$ de diciembre de 2015.

Rusia. Tribunal Constitucional. Sentencia n. ${ }^{\circ} 26-\mathrm{P}$ del 3 de julio de 2019.

Rusia. Tribunal Constitucional. Auto n. ${ }^{\circ} 20-\mathrm{O}$ del 19 de marzo de 1997.

Rusia. Tribunal Constitucional. Auto n. ${ }^{\circ}$ 171-O-P del 3 de abril de 2007.

Rusia. Tribunal Constitucional. Auto n. ${ }^{\circ} 663-\mathrm{O}$ del 28 de marzo de 2017.

Rusia. Tribunal Constitucional. Auto n. $1568-\mathrm{O}$ del 25 de junio de 2017. 